\title{
Interglaziale Klimaschwankungen in Nordost-Polen - palynologische und isotopengeochemische Untersuchungen an organischen Seesedimenten
}

\author{
Jerzy Nitychoruk, Andrzej Ber, Jochen Hoefs, Tomasz Krzywicki, Jürgen Schneider \& Hanna Winter*)
}

Nitychoruk, J., Ber, A., HOefs, J., Krzywicki, T., SCHNEIDER, J. \& WinTER, H. (2000): Interglaziale Klimaschwankungen in Nordost-Polen - palynologische und isotopengeochemische Untersuchungen an organischen Seesedimenten. - Eiszeitalter und Gegenwart, 50: 86-94; Hannover 2000.

Keywords: Quaternary, paleoclimate, Augustovian Interglacial, palynology, stable O- and C-Isotopes, Poland.

Kurzfassung: Die Ergebnisse von $\partial 18 \mathrm{O}-$ und $\partial 13 \mathrm{C}-$ Bestimmungen an Seesedimenten aus dem Augustovian-Interglazial in Nordost-Polen lassen sich nicht einfach interpretieren, weil die $218 \mathrm{O}-$ Werte für palynologisch kühle Perioden hoch $(-4$ bis $-6 \%$ ), dagegen für einen palynologisch wärmeren Zeitraum niedriger $(-8$ bis $-10 \%$ sind. Zusätzliche malakologische, palynologische und Isotopen-Untersuchungen weisen auf einen hohen Grad der Umlagerung älterer Sedimente vor allem in kühlen Perioden mit niedrigem Wasserstand hin.

Die Annahme, dass allochthoner Kalzit aus tertiären marinen Sedimenten in die Sedimentationsbecken gelangte, ermöglicht eine plausible Interpretation der Isotopen-Kurven. Kühle Perioden sind charakterisiert durch erhöhte Erosion infolge Verringerung der Vegetation. Dadurch wurde allochthoner Kalzit mit hohen 218O- und $213 \mathrm{C}$-Werten in das Becken transportiert. Warme Perioden dagegen sind durch eine reduzierte Redeposition von älteren Sedimenten gekennzeichnet, deshalb sind die Isotopen-Werte negativer. Aus dem Verlauf der Isotopen-Kurven kann deshalb nicht direkt auf Klimaänderungen, wohl aber indirekt auf klimatisch bedingt erhöhte bzw. erniedrigte Erosionsraten im Liefergebiet geschlossen werden.

*) Anschriften der Verfasser: Dr. J. Nitychoruk, Institute of Geology, Warsaw University, Al. żwirki i Wigury 93, 02-089 Warsaw, Poland, - Alexander-von-HumboldtStipendiat Göttingen; Dr. A. Ber, Polish Geological Institute, Rakowiecka 4, 00-950 Warsaw, Poland; Prof. Dr. J. Hoefs, Institut für Geochemie, Universität Göttingen, Goldschmidtstraße 1, D-37077 Göttingen, Germany; Dipl.-Geol. T. KrzYwicki, Polish Geological Institute, Rakowiecka 4, 00-950 Warsaw, Poland; Prof. Dr. J. SchNeIDER, Institut für Geologie und Dynamik der Lithosphäre, Universität Göttingen, Goldschmidtstraße 3, D-37077 Göttingen, Germany, jschnei@gwdg.de; Dr. H. WINTER, Polish Geological Institute, Rakowiecka 4, 00-950 Warsaw, Poland

\author{
[Interglacial climatic changes \\ in northeastern Poland - pollen, isotopes and \\ geochemical investigation of organic \\ lake sediments]
}

Abstract: $\partial 18 \mathrm{O}$ and $\partial 13 \mathrm{C}$ values were examined from Quaternary lake sediments of the Augustovian Interglacial (Tab.1) in northeastern Poland. The results cannot be easily interpreted, because values of $\partial 18 \mathrm{O}$ are high $(-4$ to $-6 \%$ ) for cool intervals (according to palynological analysis) and quite low (-8 to $-10 \%$ ) for warmer intervals. Supplementary mollusc, pollen and isotope examination indicated the presence of a considerable redeposition of older sediments during lower lake levels mostly during the cool intervals. Assumption that allochthonous calcite from Tertiary marine sediments was transported into the lake basin, allows a reasonable interpretation of the isotope curves. Cool intervals indicate more intensive erosion due to less dense vegetation. Allochthonous older calcite (with high values of $\partial 18 \mathrm{O}$ and $213 \mathrm{C}$ ) therefore was transported into the lake. On the other hand, warm intervals are characterised by reduced redeposition of older sediments, and therefore values of $218 \mathrm{O}$ are more negative. Although the isotope curves do not permit to draw direct conclusions on temperature and climatic changes, they indicate more or less intensive erosion rates in the area around the lake.

\section{Einleitung}

Im nord-östlichen Polen erreichen die quartären Sedimente eine Gesamt-Mächtigkeit von mehr als $200 \mathrm{~m}$. Bohrungen, welche die Sedimente vollständig durchteuft haben (Abb. 1), stießen in den tieferen Abschnitten von vier Bohrkernen auf See- und Torfsedimente mit einer Mächtigkeit von 4,6 bis 31,1 m. Diese Ablagerungen sind gekennzeichnet durch Torfe, Gyttjen, Tone, Silte und fein- bis grobkörnige Sande, die stellenweise Süßwasserschnecken enthalten (KRZYwICKI \& LISICKI 1993, Ber \& LISICKi 1995, Ber 1989, 1996, 1999a, b, Ber et al. 1998).

Die fossilen Seesedimente wurden palynologisch, malakologisch, paläomagnetisch und geochemisch untersucht und mit Hilfe der Thermolumineszenz-Methode datiert (Ber 1996, 1999a, b, Ber et al. 1998, s. Tab. 1). 


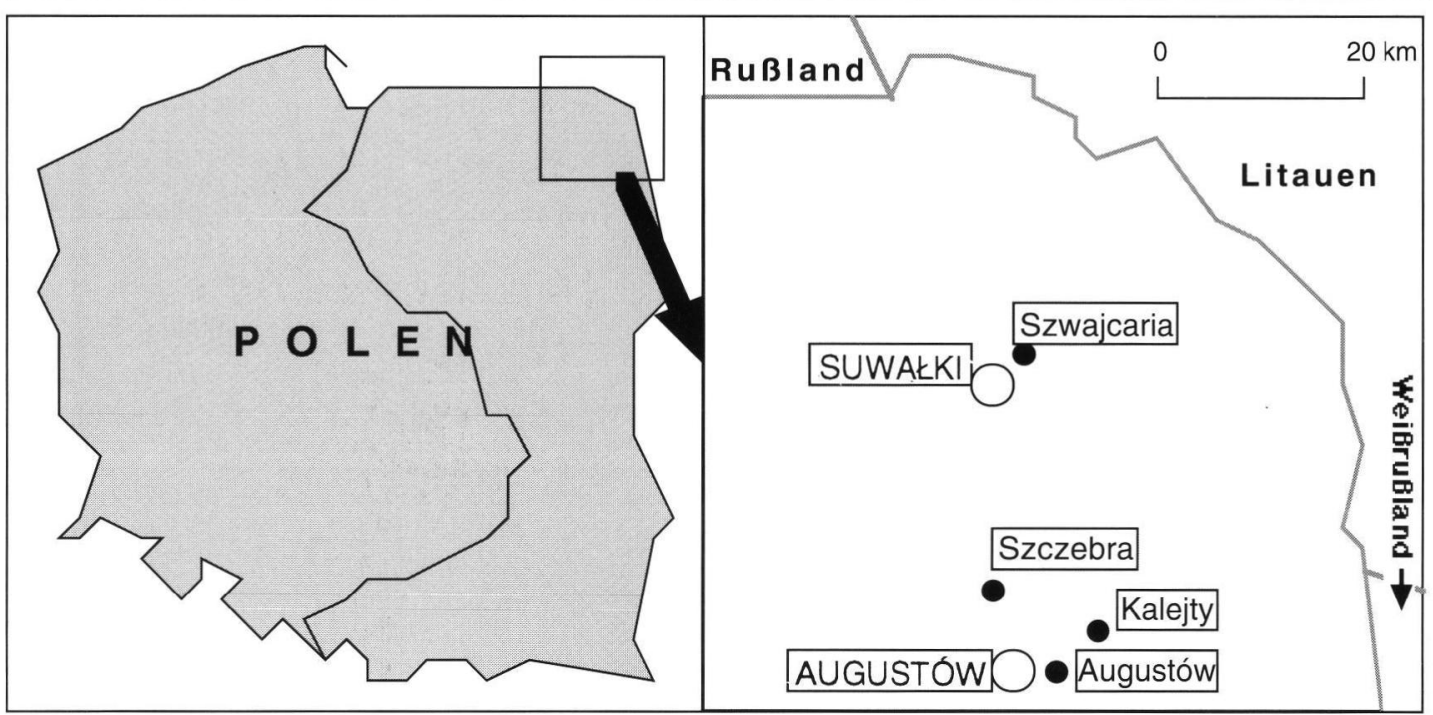

Abb. 1. Lokalitäten mit interglazialen Sedimenten im nordöstlichen Polen.

Fig. 1. Studied sites with interglacial sediments in northeastern Poland.

Die bisherigen geologischen Untersuchungen haben ergeben, dass das Gebiet, in dem die lakustrinen Serien nachgewiesen wurden, im Quartär eine Senke bildete. In diesem Seebecken wurden teils fluviale, teils Flachwassersedimente abgelagert (BER 1996). Die Seesedimente lagern auf Geschiebelehmen und fluvioglazialen Sanden aus der ältesten Vereisung des Narevian (Tab. 1) und

Tab. 1. Zwei mögliche chronostratigraphische Positionen der Augustovian-Stufe in Polen nach Ber et al. (1998). Der schwarz/weiße Balken kennzeichnet die paläomagnetischen Epochen.

Tab. 1. Two possible chronostratigraphic positions of the Augustovian stage in Poland after Ber et al. (1998). The black/white column indicates the paleomagnetic stages.

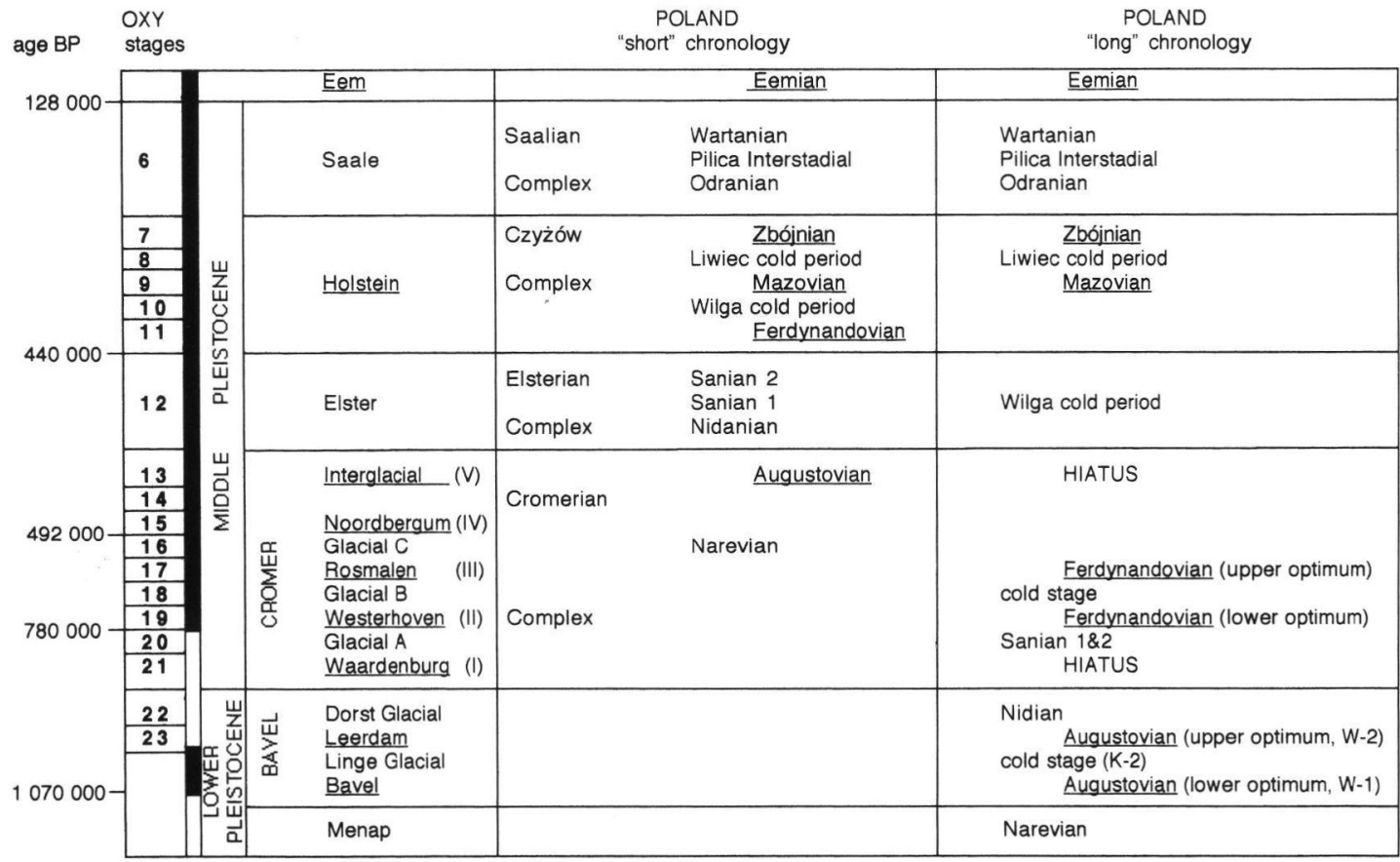


[m über NN]

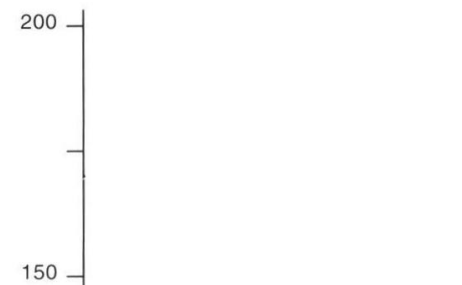

KALEJTY

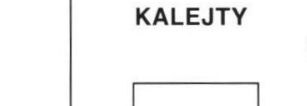

SZCZEBRA

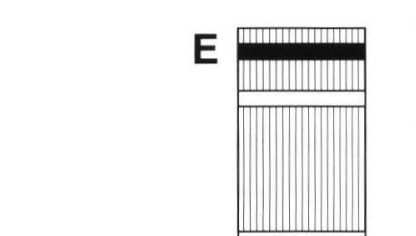

Augustów

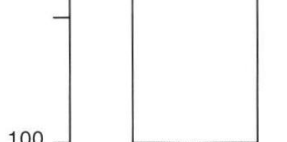

100
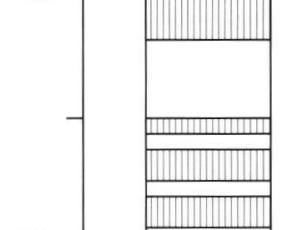

$$
50
$$

5

50







-50
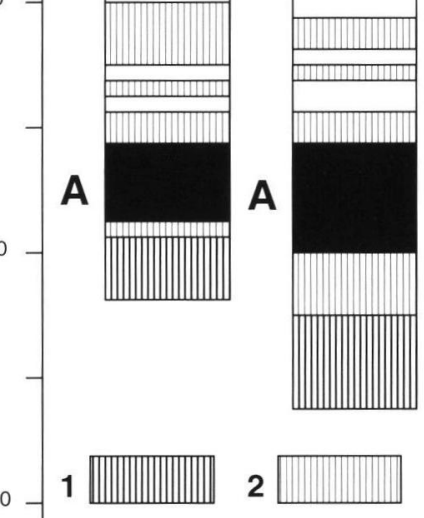

$3 \mathbf{H}$

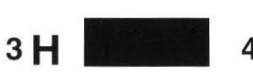

Abb. 2. Zusammenstellung der quartären Schlüsselprofile der Bohrkerne aus dem nordöstlichen Polen (nach Ber 1996, vereinfacht).

1 - Mergel des unteren Paläozäns; 2 - Geschiebelehm; 3 - interglaziale organische Sedimente; 4 - andere glaziale Sedimente, A - Augustovian-Stufe; H - Holstein-Interglazial; E - Eem-Interglazial.

Fig. 2. Quaternary key columns of cores from Northeastern Poland - simplified, after BER (1996).

1 - Palaeocene marl; 2 - till; 3 - interglacial organic sediments; 4 - other glacial deposits, A - Augustovian Interglacial; H - Holsteinian Interglacial; E Eemian Interglacial.

sind von einem mächtigen glazialen Komplex überdeckt, der u. a. aus Geschiebelehm sowie interglazialen Sedimenten besteht. Darüber liegen See-Sedimente aus dem interglazialen Ferdynandovian (Westerhoven), dem Mazovian (Holstein) und dem Eem (Abb. 2, Tab. 1).

Palynologische Untersuchungen, die an zwei Bohrkernen (Szczebra und Kalejty, Abb. 3 und 4) aus den neu entdeckten fossilen Seesedimenten durchgeführt wurden, erlauben es, eine neue Stufe in der Klimastratigraphie Nordost-Polens nachzuweisen. Diese Stufe wird nach JaNCZIK-Kopikowa (1996) als Augustovian-Interglazial bezeichnet.

Charakteristische Merkmale dieses Interglazials im Profil Kalejty sind zwei warme (W-1 und W-2) und drei kühle Perioden (K-1, K-2, K-3, Abb. 3 und 4). Die ältere warme Periode (W-1) zeichnet sich durch eine boreale Waldflora (in Kalejty und Szczebra) aus sowie durch eine Wasserflora mit Azolla filiculoides (in Szczebra). Beide sprechen für ein warmes Klima (JANCZYK-KoPIKOWA 1996). Die jüngere warme Periode (W-2) zeigt eine typische interglaziale Flora, die für ein gemäßigt warmes Klima charakteristisch ist (Abb. 4). Die ältere warme Periode (W-1) zeigt palynologisch eine geringere Erwärmung als die jüngere (W-2), die von JANCZYK-KOPIKOWA (1996) mit den warmen Phasen des Pastonian in England (GIBBARD et al. 1991, West 1996) und des Leerdam-Interglazials in Holland (ZAGWIJN \& DE JONG 1984) korreliert wird. Die Pollensukzession des Augustovian-Interglazials nach JANCZYK-KOPIKOWA (BER et al. 1998) unterscheidet sich deutlich von der Pollensukzession des Cromerian-Interglazials in Holland (ZAGWIjN 1996) und von dem "Cromerian Complex" in Deutschland (Müller 1992). Die warmen klimatischen Perioden sind durch kühle Phasen getrennt (K-1, K-2, K-3), in denen ein subarktisches Klima herrschte.

Die oben dargestellten Untersuchungsergebnisse erlauben es noch nicht, die stratigraphische Position des Augustovian-Interglazials genau zu bestimmen.

Die von JaNCZYK-KopIKOWa (1996) vorgenommene Zuordnung zum Bavel bzw. die von Ber et al. (1998) bzw. BER (1999 a, b) vorgenommene Zuordnung zum Cromer-Komplex verlangt weitere Untersuchungen, die zur Zeit durchgeführt werden. 


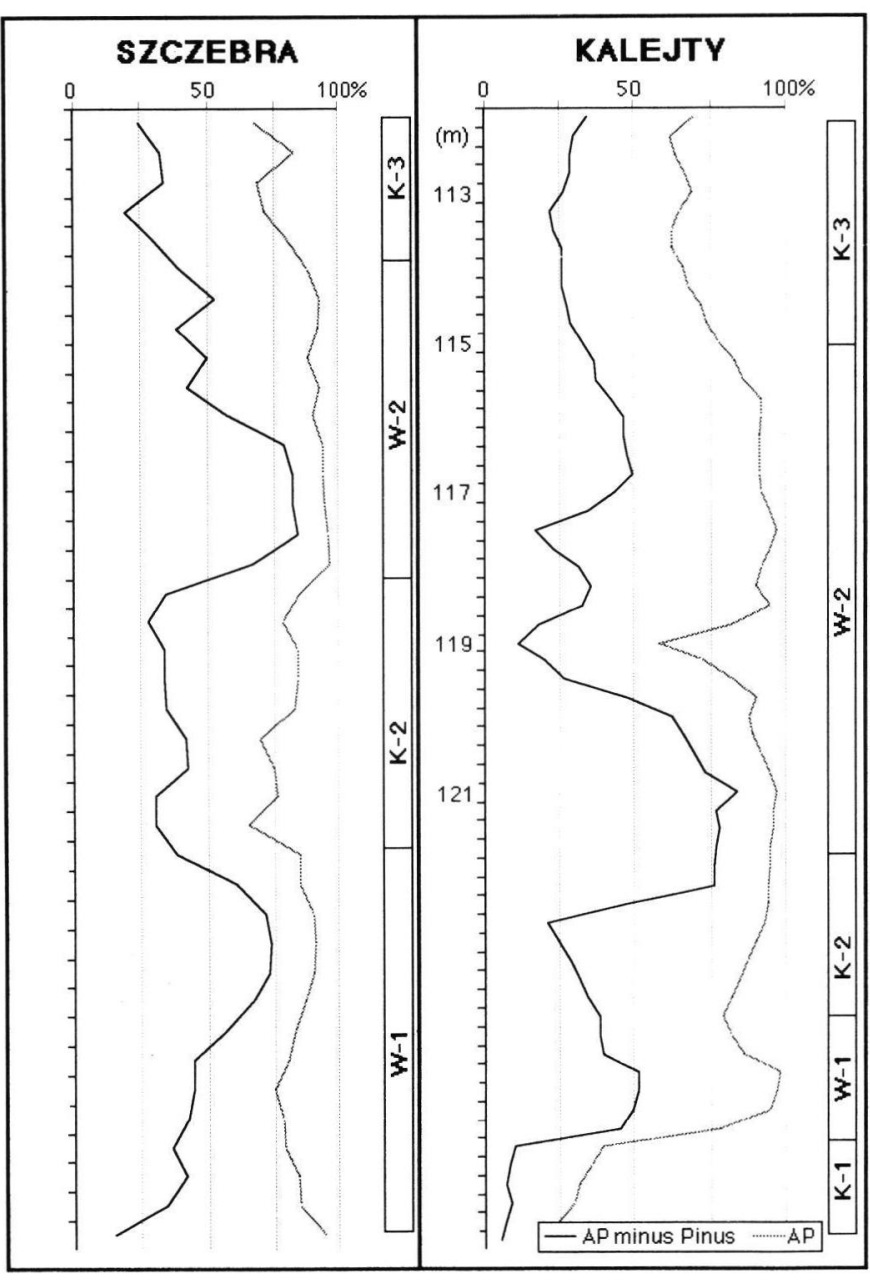

Abb. 3. Summe des Baumpollens minus Pinus (AP minus Pinus) und des Baumpollens (AP) für die Lokalitäten Kalejty (Analysen von $\mathrm{H}$. WinTER, Abb. 4) und Szczebra nach JANczYK-KopIkowa (1996).

Fig. 3. Sum of tree pollen minus Pinus (AP minus Pinus) and tree pollen (AP) for Kalejty (analyses: H. WinTer, Fig. 4) and Szczebra according to JANCZYK-KOPIKOWA (1996).

\section{Sedimentbeschreibung}

Die Seesedimente im Profil Kalejty bestehen vorwiegend aus Silt mit eingeschalteten Tonen, sowie aus feinkörnigem und siltigem Sand. In den Teilen des Bohrkerns, in denen warme Klimaperioden palynologisch festgestellt wurden, treten außerdem in der ersten Klima-Periode (W-1) Pflanzenreste und schwarzer humoser Sand mit gut erhaltenen Insekten auf, in der jüngeren Klima-Periode (W-2) Pflanzenreste und SüßwasserSchnecken und -Muscheln. Die Ablagerungen enthalten auch nennenswerte Mengen an Muskovit und Glaukonit. Besonders häufig sind diese Minerale in den Abschnitten zu finden, deren Se- dimentation unter kühlen klimatischen Verhältnissen erfolgte.

\section{Analyse-Methoden}

Für die Bestimmung der stabilen $\mathrm{O}$ und C-Isotope wurden bisher aus den Bohrkernen von Kalejty aus einer Tiefe zwischen 107,0 und 130,0 47 Proben im Abstand von je einem halben Meter entnommen.

Der $\mathrm{CaCO}_{3}$-Gehalt der Seesedimente von Kalejty (Abb. 5) ist im allgemeinen gering (Analyse mit Scheibler). Der geringe Karbonatgehalt der Sedimente aus der ersten warmen Periode W-1 $(124,5-126,5 \mathrm{~m})$ und dem Beginn der dritten kühlen Periode K-3 (115,0$119,5 \mathrm{~m}$ ) unmittelbar nach dem zweiten warmen Abschnitt lässt Isotopenbestimmungen für diese Kernteile nicht sinnvoll erscheinen. Die Zusammensetzung der Sauerstoff- und Kohlenstoff-Isotope wurde mit der klassischen Methode nach McCrEa (1950) bestimmt.

Das durch Reaktion mit 99\%iger Orthophosphorsäure gewonnene $\mathrm{CO}_{2}$ wurde mit einem Massenspektrometer (Finningan-Mat 251) im Geochemischen Institut der Universität Göttingen analysiert. Die $18 \mathrm{O}$ - und $13 \mathrm{C}$-Konzentrationen in den untersuchten Proben wurden als $\partial$-Werte im Vergleich zum PDB-Standard ausgedrückt. Der analytische Fehler für den Wert $\partial 18 \mathrm{O}$ beträgt $\pm 0,2 \%$ und für $\partial 13 \mathrm{C} \pm 0,1 \%$.

\section{Isotopenbestimmungen}

\section{d18 O-Kurve (Abb. 5)}

In der ersten kühlen Periode (K-1) der Augustovian-Stufe im Profil Kalejty schwankt der durch den d18O-Wert ausgedrückte O-Isotopen-Gehalt in den Sedimenten von $-4,3$ bis $-9,1 \%$. Die höchsten negativen $\partial 18 \mathrm{O}$-Werte $(-9,1 \%)$ wurden an Sedimenten mit größerem Anteil an organischem Material gemessen. Der weitere Kernteil aus der Abkühlungszeit K-1 zeigt positivere $\partial 18 \mathrm{O}$-Werte, was bei Sedimenten aus kontinentalen Seen ein Indikator für warme Klimaverhältnisse ist (EICHER \& Siegenthaler 1976, 1981, McKenzie \& Eberli 1987, Nitychoruk et al. 1999, Nitychoruk 2000). Im vorliegenden Fall steht diese Deutung aller- 


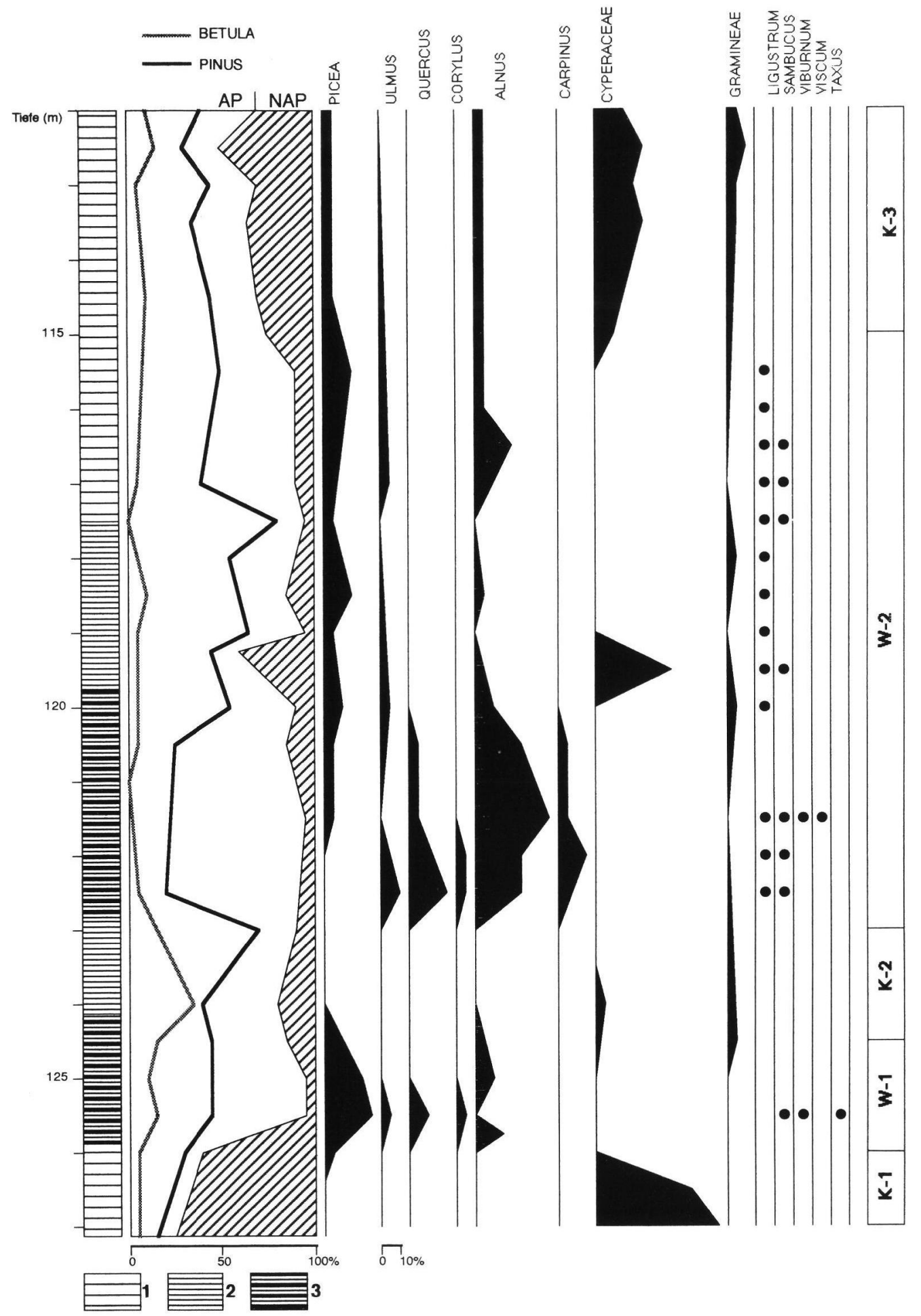

Abb. 4. Pollendiagramm des Augustovian-Interglazials für die Lokalität Kalejty.

1 - Silt, 2 - Ton, 3 - Silt, Sand und Ton mit Süßwasserfauna, Insekten und Pflanzenresten.

Fig. 4. Pollen diagram of the Augustovian Interglacial from Kalejty.

1 - silt, 2 - clay, 3 - silt, sand and clay with molluscs, insects and organic detritus. 
dings für den oberen Teil von K-1 im Widerspruch zu den palynologischen Untersuchungen, aus denen hervorgeht, dass das Klima während der gesamten Phase K-1 kühl war.

Die erste warme Periode (W-1), die der Phase $\mathrm{K}-1$ folgt, ist in karbonatfreien Sand-Ablagerungen mit organischem Material dokumentiert. Daher liegen aus diesem Abschnitt keine Isotopendaten vor. Die nächste kühle Periode (K-2) ist durch allmählich von $-8,3$ bis auf $-8,8 \%$ sinkende d 18 O-Werte gekennzeichnet. Solche Werte sind typisch für Seesedimente aus kühlen PleistozänPerioden (Nitychoruk et al. 1999, NitychORLK 2000).

Die Klima-Abkühlung zeigt sich deutlich im palynologischen Bild. Der Prozentanteil wärmeliebender Bäume, wie Eiche, Ulme und Hasel sinkt zugunsten von Birke und Kiefer, die kühle klimatische Bedingungen besser ertragen (Abb. 4).

Die zweite warme Periode (W-2) wurde auf Grund der palynologischen Untersuchungen (JANCZYK-KopIKOWA 1996) als die wärmste Zeit dieses Interglazials bestimmt. Wenn man jedoch das für diese Periode gewonnene Isotopenbild mit vorangehenden klimatischen Phasen ver-

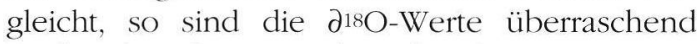
niedrig $(-7,2$ bis $-9,0 \%)$ und nicht repräsentativ für das palynologisch bestimmte klimatische Interglazial-Optimum. Für den jüngeren Teil aus der warmen Periode (W-2) liegen keine Isotopenbestimmungen vor, da keine Karbonate auftreten.

Die Abkühlungsperiode $\mathrm{K}-3$ ist durch hohe $\partial 18 \mathrm{O}-$ Werte von $-3,6$ bis $-7,9 \%$ charakterisiert, die ebenfalls im Widerspruch zu dem sich aus den palynologischen Untersuchungen ergebenden kühlen Klima stehen. Die Zunahme des prozentualen Anteils an $\mathrm{CaCO}_{3}$ in den Sedimenten während der Abkühlungsphasen K-1 bis K-3 geht mit einer Verschiebung der $\partial 18 \mathrm{O}$-Kurve in Richtung höherer Werte einher (Abb. 5).

\section{ว13C-Kurve (Abb. 5)}

Die niedrigsten $\partial 13 \mathrm{C}$-Werte (bis $-8,5 \%$ ) wurden für den Kernteil ermittelt, der im klimatischen Optimum (Augustovian, Erwärmung W-2) entstand. Mit Ausnahme dieser Werte verläuft die d13C-Kurve ziemlich gleichmäßig und zeigt Werte zwischen 0,9 und $-1,8 \%$.

In der ersten und der dritten kühlen Periode, K-1 und K-3, zeigt sich eine schwache Korrelation Zwischen den $\partial 18 \mathrm{O}-$ und $\partial 13 \mathrm{C}-K u r v e n$, was typisch für Karbonate in hydrologisch abgeschlossenen
Seebecken ist. Diese Korrelation ist in der kühlen Phase K-2 und der warmen Phase W-2 nicht vorhanden, was als Indikator für hydrologisch offene Seen interpretiert werden kann (EICHER \& SiEGENthaler 1976, Fritz et al. 1975, TAlbot 1990)

Der beschriebene Verlauf der Isotopen-Kurven ist für kontinentale Seen nicht charakteristisch. Die negativeren $\partial 18 \mathrm{O}$-Werte in einer warmen Periode und höhere in einer kühlen Periode sind allerdings typisch für marine Ablagerungen. Dieses unerwartete Ergebnis erforderte weitere Untersuchungen, die eine plausible Rekonstruktion des Ablagerungsmilieus aus dem Augustovian-Interglazial ermöglichten.

\section{Fauna}

Die Sedimente von Kalejty enthalten marines Plankton aus der Gruppe der Dinoflagellaten. In dem benachbarten Bohrkern Szczebra (Abb. 1) wurde kein marines Plankton in der AugustovianStufe festgestellt. Daraufhin wurde die Süsswasserfauna aus der warmen Periode W-2 aus dem Bohrkern in Kalejty untersucht. Dabei stellte sich heraus, dass zwei dort gefundene Schnecken, Valvata naticina und Gyraulus albus, sowie eine Muschel, Pisidium moitessierianum, nicht zur marinen, sondern zur Süßwasser-Fazies zuzurechnen sind, mit deren Hilfe man das Ablagerungsmilieu der betreffenden Sedimente beschreiben kann (SKOMpski 1991). Die Gastropode Valvata naticina (MENKE) lebt vor allem in langsam fließenden Flüssen. Seltener besiedelt sie auch kleinere Flüsse und Seen. Sie kommt in Europa von der Elbe bis zum Dnjepr vor. Die Gastropode Gyraulus albus (MüLLER) bevorzugt stehende Gewässer, sie ist aber auch in fließenden Gewässern anzutreffen. Pisidium moitessierianum (PALADIHLE) lebt sowohl in Seen und Flüssen als auch in kleinen Seen, sie vermeidet schnell fließende Gewässer (Sкомpski 1991).

Die beschriebene Süsswasserfauna spricht für einen langsam durchflossenen See, der von einem Fluss gespeist wurde. Eine ähnliche Faunen- $\mathrm{Zu}-$ sammensetzung mit Valvata naticina und Pisidium moitessierianum wurde in den fossilen Sedimenten des Drużno-Sees aus dem Holozän (südlich von Gdarisk) nachgewiesen, in dem sich bis vor etwa 1000 Jahren das Delta der Weichsel aufschüttete (NITYCHORuK 1998).

\section{Flora}

Im Zuge der palynologischen Untersuchungen wurde auch umgelagerter Pollen aus dem Tertiär 


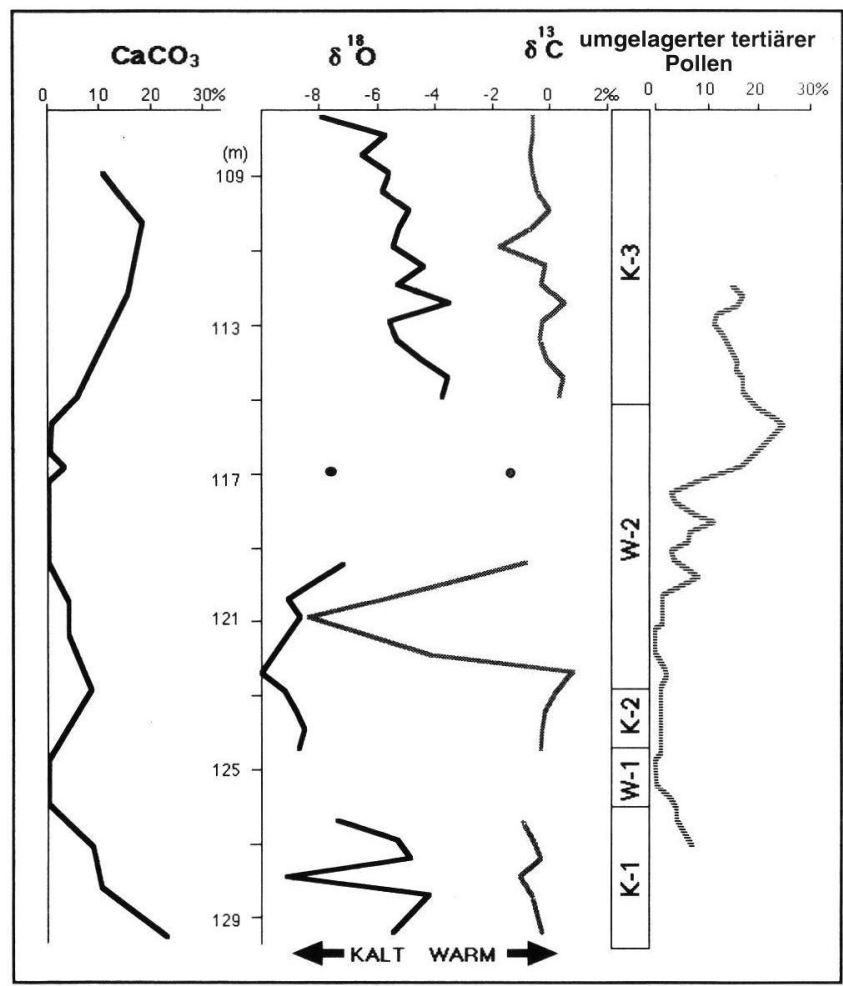

Abb. 5. Isotopen-Kurven und prozentualer Gehalt des umgelagerten tertiären Pollens aus dem Augustovian-Interglazial in Kalejty. (Angaben für Kalt bzw. Warm nach den palynologischen Ergebnissen)

Fig. 5. Stable isotope records and redeposited Tertiary pollen from the Augustovian Interglacial at Kalejty. (Cold - K, resp. Warm - W, after palynological results).

nachgewiesen (Abb. 5). Der geringste Pollenanteil wurde in den warmen Perioden W-1 und W-2 nachgewiesen, die größte Menge tritt in den kühlen Perioden K-1 und K-3 auf (bis zu 30\% des Gesamtpollenanteils). So ein hoher Anteil an umgelagertem Pollen spricht für eine allochthone Zufuhr von Material in das Gewässer in Phasen niedrigeren Wasserstandes. Für Erosion und Resedimentation sprechen auch der detritische Muskovit, Glaukonit und die marinen Karbonate, die in den Ablagerungen gefunden wurden.

\section{Zusätzliche Isotopenuntersuchungen}

An der Süßwasserfauna und den siltig/sandigen Einzel-Lagen mit einer Mächtigkeit von $0,5 \mathrm{~cm}$ aus der warmen Periode W-2, wurden zusätzliche Isotopenbestimmungen durchgeführt. Die karbonatische Sandfraktion und Schneckengehäuse besitzen ähnliche $218 \mathrm{O}$-Werte $(-6,9$ und $-6,4 \% 0)$. Diese Werte sind positiver als die $218 \mathrm{O}-$ Werte der karbonatischen Silte und der Muscheln (-9,1 und
$-8,8 \%$ ). Dies wurde an fünf Proben beobachtet und erlaubt folgende Annahme: Sandige Lagen weisen auf einen Transport durch stärkere Strömung hin. Ebenso können Schneckengehäuse über größere Entfernungen transportiert werden, dabei kann Faulgas oder Luft, die sich in den Gehäusen ansammeln, die Schalen im Wasser schwebend verdriften. Die Muschelschalen werden dagegen nicht so weit transportiert.

Die Sedimente können demnach in einem Fluss transportiert worden sein, bevor sie zur endgültigen Ablagerung in ein ruhigeres Becken kamen. Ob es sich bei der Wechsellagerung sandig/siltig um nichtglaziale Warven oder durch wechselnde Transportgeschwindigkeit des Wassers bedingte Korngrößen-Unterschiede handelt, ist unklar.

Diese Interpretation des Ablagerungsmilieus lässt den Schluss zu, dass bei der Sedimentation in Kalejty im Augustovian-Interglazial der Materialtransport zum Ablagerungsort eine wichtige Rolle spielte. Der Ablagerungsort könnte der Bereich vor der Deltamündung eines Flusses sein, der in einen See floss.

Der allochthone Materialtransport hat einen wesentlichen Einfluss auf die gemessenen Isotopenwerte. Wenn es möglich war, dass zusammen mit Muskovit, typisch marinem Glaukonit und Sporomorphen von Tertiär-Pflanzen auch marines Plankton und ganze Schneckengehäuse transportiert wurden, sollte auch das in den Sedimenten enthaltene Karbonat wahrscheinlich zum großen Teil allochthoner Herkunft sein.

Ein höherer Gehalt an $\mathrm{CaCO}_{3}$ in kühlen Phasen des Augustovian bedeutet, dass es infolge einer Lichtung der Pflanzendecke zu erhöhter Erosion und zur Zufuhr allochthonen Kalzits gekommen sein könnte. Die das Karbonat transportierende Wasserströmung stammte höchstwahrscheinlich von einem größeren Fluss. Die Karbonat-Quellen können in den paläozänen Mergeln und/oder dem Geschiebelehm vermutet werden, der die Augustovian-Sedimente unterlagert. Die an tertiären Mergeln durchgeführten Isotopenbestimmungen brachten als Ergebnis: $\partial 18 \mathrm{O}$ bis $-1,6 \%$, $\partial 13 \mathrm{C}$ bis $+0,6 \%$, was die hohen $\partial$-Werte für kühle Phasen erklären kann. 
Obwohl man aus den vorliegenden Isotopenkurven keine direkten Schlussfolgerungen auf Klima und Temperatur ziehen kann, erlauben sie doch eine Aussage zu den Palaeoumwelt-Bedingungen. Eine wichtige Information, die man dem Kurvenverlauf entnehmen kann, ist der in den kälteren Phasen festgestellte hohe Anteil an allochthonem Kalzit in den Sedimenten. Dies spricht für eine verstärkte Erosion älterer Sedimente und für eine auch palynologisch nachweisbare Verringerung der Pflanzendecke.

Der Einfluss allochthoner Karbonate auf die Isotopenzusammensetzung der Seesedimente bezieht sich vorwiegend auf die kühlen Perioden K-1 und K-3 der Augustovian-Stufe. Die kühle Periode $\mathrm{K}-2$ und die warme Periode $\mathrm{W}-2$ sind charakterisiert durch stärker negative $218 \mathrm{O}$-Werte, die nicht auf einen erheblichen Anteil an allochthonem Kalzit hindeuten. Auch die Menge des umgelagerten Pollens aus dem Tertiär verringerte sich in dieser Zeit. Das Fehlen der $\partial^{18} \mathrm{O}-$ und $\partial^{13} \mathrm{C}-$ Korrelation für die warme Periode W-2 sowie die Verschiebung der Isotopenkurven in Richtung negativer Werte sprechen für hydrologische Verhältnisse im See, die einem offenen System zugeordnet werden können (EICHER \& Siegenthaler 1976, Fritz et al. 1975).

Die stärker negativen Werte für die d13C-Kurve (W-2 in Abb. 5) sind wahrscheinlich auf einen erhöhten Anteil an biogenem $\mathrm{CO}_{2}$ zurückzuführen, das durch die palynologisch nachweisbare Klimaerwärmung und die daraus resultierende höhere Bioproduktion und erhöhten Abbau organischer Substanz bedingt war (FrITz \& POPLAwSKI 1974, JEDRYSEK 1994, WaChNIEW \& RoŻAÑSKI 1998).

Die kühlen Perioden K-1 und K-2 sind durch hohe Isotopenwerte charakterisiert und durch eine vergleichsweise gute Korrelation zwischen $\partial 18 \mathrm{O}$ und $\partial^{13} \mathrm{C}$ (in K-1), was bedeuten kann, dass der Seespiegel niedriger und der See hydrologisch geschlossen war. Die Erosion und der Transport präquartärer Sedimente erfolgte wahrscheinlich in dem Zufluss während der kühlen und niederschlagsärmeren Perioden

\section{Schlussfolgerungen}

Die Interpretation der Isotopenkurven für die Sedimente aus Kalejty stimmt nicht mit den palaeobotanisch rekonstruierten Klimaveränderungen überein. Sie können jedoch als ein Indikator für Erosion und Umlagerung von älteren Sedimenten sowie für hydrologische Veränderungen im Sedimentationsbecken genutzt werden.
Es ergibt sich weiterhin, dass

- das Becken, in dem die Sedimentation erfolgte, zeitweise durch den Eintrag aus einem Fluss beeinflusst wurde,

- das gleichzeitige Vorkommen von Süßwasserfauna und marinem Plankton nicht für marine Bedingungen spricht. Das schließt die Interpretation der Isotopendaten aus den Sedimenten als marine Ablagerungen aus,

- der große Anteil tertiären Pollens an umgelagerter Süsswasserfauna und an allochthonen Mineralen wie Muskovit und Glaukonit im Sediment ein Indiz dafür ist, dass auch der im Sediment vorkommende Kalzit wohl zum großen Teil allochthon ist (Ausnahme: Phase W-2).

\section{Dank}

Wir danken Herrn Dr. Josef MerkT (Niedersächsisches Landesamt für Bodenforschung) und Herrn Prof. Dr. Bernd Zolitschka (Institut für Geographie der Universität Bremen) für die hilfreiche kritische Durchsicht des ursprünglichen Manuskriptes.

Der Alexander-von-Humboldt-Stiftung dankt der Erstautor (Dr. J. NitychORuk) herzlich für die Gewährung eines eineinhalbjährigen Stipendiums (Az. POL/1020220) in der Arbeitsgruppe Limno-/ Umweltgeologie am Institut für Geologie und Dynamik der Lithosphäre der Universität Göttingen.

\section{Schriftenverzeichnis}

BER, A. (1989): Stratigraphy of the Quaternary of the Suwalki Lakeland and its substrate based on recent data. - Geological Quarterly, 38: 149-165; Warszawa,

- (1996): Geological situation of Augustovian (Pastonian) Interglacial lake sediments at Szczebra near Augustow and Mazovian Interglacial organogenic sediments at Krzyżewo. - Biuletyn Panstwowego Instytutu Geologicznego, 373: 35-48; Warszawa.

- (1999a): Plejstocen Polski NE w nawiazaniu do głe,bokiego podtoża. - Prace Państwowego Instytutu Geologicznego; Warszawa (in Druck).

- (1999b): The Middle-Lower Pleistocene stratigraphy in the northeastern Poland. - XV International Congress INQUA, 3-11 August 1999, Durban, South Africa, book of abstracts: 21; Durban.

- JANCZYK-KOPIKOWA, Z. \& KRZYSZKOWSKI, D. (1998): A new interglacial stage in Poland (Augustovian) and the problem of the age of the oldest pleistocene till. - Quaternary Science Reviews, 17: 761-773; Washington. 
- \& Lisicki, S. (1995): Stratygrafia plejstocenu NE Polski (pojezierza Mazurskie i Suwalskie oraz Ròwnina Augustowska) w swietle nowych danych. - II Konferencja „Stratygrafia Plejstocenu Polski“. Grabanòw 18-20 wrzesnia 1995; Grabanòw.

Eicher, U. \& Siegenthaler, U. (1976): Palynological and oxygen isotope investigations on Late-Glacial sediment cores from Swiss lakes. - Boreas, 5: 109-117, Oslo.

- Sifgenthaler, U. \& Wegmüller, S. (1981): Pollen and Oxygen Isotope Analyses on Late- and PostGlacial Sediments of the Tourbiere de Chirens (Dauphiné, France). - Quaternary Research, 15: 160-170; Washington.

Fritz, P., ANderson, T. W. \& Lewis, C. F. M. (1975): LateQuaternary Climatic Trends and History of Lake Erie from Stable Isotope Studies. - Science, 190: 267-269; Washington.

- \& Poplawski, S. (1974): 180 and $13 \mathrm{C}$ in the shells of freshwater molluscs and their environments. - Earth and Planetary Science Letters, 24: 91-98; Amsterdam.

Gibbard, P. L., West, R. G., ZaGwijn, W. H. \& 14 Autoren (1991): Early and early Middle Pleistocene correlations in the southern North Sea Basin. - Quaternary Science Reviews, 10: 23-52; Washington.

JANCZYK-KopIKowa, Z. (1996): Temperate stages of the Mesopleistocene in Northeastern Poland. - Biuletyn Pañstwowego Instytutu Geologicznego, 373: 49-66; Warszawa.

JęDryseK, M.O. 1994. Carbon isotope evidence for diurnal variations in methanogenesis in freshwater lake sediments. - In: M. O. JĘDRYsEK [ed.]: Isotope workshop II, Extended Abstracts: 66-68; Wroctaw.

KrzYwicki, T. \& Lisicki, S. (1993): Czwartorzed Pojezierza Suwalskiego. - Przew. LXIV Zjazdu Polskiego Towarzystwa Geologicznego na Ziemi Suwalskiej. 9-12 wrzesnia 1993; Warszawa.

McCrea, J. M. (1950): The isotopic chemistry of carbonates and a paleotemperature scale. - Journal of Chemical Physics, 18: 849-857; New York.

McKenzie, J. A. \& Eberli, G. P. (1987): Indications for abrupt Holocene climatic change: Late Holocene oxygen isotope stratigraphy of the Great Salt Lake, Utah. - In: W. H. Berger \& L. D. Labeyrie [eds.]: Abrupt Climatic Change: 127-136; (D. Reidel Publishing Company).

Müller, H. (1992): Climate changes during and at the end of interglacials of the Cromerian Complex. - In: KukLA, G. J. \& Went, E. [eds.]: Start of Glacial, NATO ASI Series, I 3: 51-69.
Nitychoruk, J. (1998): Podsumowanie badan geologicznych Zuław Elblaskich przeprowadzonych w ramach programu "Adalbertus". - In: URBAN̂CZYK, P. [ed.]: Adalbertus. Wyniki programu badan interdyscyplinarnych, 1: 23-34; Warszawa.

- (2000): Climate reconstruction from stable-isotope composition of the Mazovian Interglacial (Holsteinian) lake sediments in eastern Poland. - Acta Geologica Polonica, 50: 247-294; Warszawa.

- Hoefs, J. \& Schneider, J. (1999): Klima-Änderungen im Pleistozän: Isotopenuntersuchungen an fossilen Seesedimenten aus dem Holstein-Interglazial OstPolens. - Eiszeitalter und Gegenwart, 49: 21-34; Hannover.

Skомpski, S. (1991): Fauna czwartorzedu Polski - bezkregowce. - Wydawnictwa Universytetu Warszawskiego: 97 p.; Warszawa.

TAlbot, M. R. (1990): A review of the palaeohydrological interpretation of carbon and oxygen isotopic ratios in primary lacustrine carbonates. - Chemical Geology. Isotope Geoscience Section, 80: 261-279.

WACHNIEW, P. \& ROŻANSKI, K. (1998): Isotopic composition of calcite deposited in Lake Gościąż, under present climatic conditions. - In: M. RALSKA-JasIEWICZOWa, T. Goslar, T. Madejska \& L. Starkel [eds.]: Lake Gościaż, Central Poland a monographic study, part 1: 61-66; Kraków.

WEST, R. G. (1996): Outline of the stratigraphy and vegetational history of the Cromer Forest-bed Formation. In: Turner, C. [ed.]: The Early Middle Pleistocene in Europe: 1-8; Rotterdam-Brookfield (A. A. Balkema).

ZaGwiJn, W. H. (1996): The Cromerian Complex Stage of the Netherlands and correlation with other areas in Europe. - In: Turner, C. [ed.]: The Early Middle Pleistocene in Europe: 145-172; Rotterdam-Brookfield (A. A. Balkema).

- \& De Jovg, J. (1984): Die Interglaziale von Bavel und Leerdam und ihre stratigraphische Stellung im niederländischen Früh-Pleistozän. - Mededelingen Rijks Geologische Dienst, 37: 155-169; Haarlem.

Manuskript eingegangen am 7. September 1999, zum Druck angenommen am 16. Oktober 2000 